\title{
Multinational Registries: Challenges and Opportunities
}

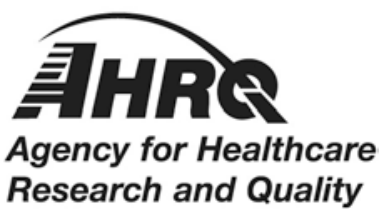




\title{
Research White Paper
}

\section{Multinational Registries: Challenges and Opportunities}

\author{
Addendum to Registries for Evaluating Patient Outcomes: A \\ User's Guide, Third Edition
}

\section{Prepared for:}

Agency for Healthcare Research and Quality

U.S. Department of Health and Human Services

5600 Fishers Lane

Rockville, MD 20857

www.ahrq.gov

Contract No. 290-2014-00004-C

Prepared by:

L\&M Policy Research, LLC

Washington, DC

Author:

Michelle B. Leavy, M.P.H.

OM1

AHRQ Publication No. 17(18)-EHC016-EF

February 2018 
The Effective Health Care Program of the Agency for Healthcare Research and Quality (AHRQ) conducts and supports research focused on the outcomes, effectiveness, comparative clinical effectiveness, and appropriateness of pharmaceuticals, devices, and health care services. More information on the Effective Health Care Program can be found at www.effectivehealthcare.ahrq.gov.

This report is based on research conducted by L\&M Policy Research, LLC, with partners OM1 and IQVIA, under contract to the Agency for Healthcare Research and Quality, Rockville, MD (Contract No. 290-2014-00004-C). The findings and conclusions in this document are those of the authors, who are responsible for its contents; the findings and conclusions do not necessarily represent the views of AHRQ. Therefore, no statement in this report should be construed as an official position of AHRQ or of the U.S. Department of Health and Human Services.

\section{None of the authors have any affiliations or financial involvement that conflicts with the material presented in this report.}

This report may be used and reprinted without permission except those copyrighted materials that are clearly noted in the report. Further reproduction of those copyrighted materials is prohibited without the express permission of copyright holders.

Persons using assistive technology may not be able to fully access information in this report. For assistance contact EPC@ahrq.hhs.gov.

Suggested citation: Leavy MB. Multinational Registries: Challenges and Opportunities. White Paper, addendum to Registries for Evaluating Patient Outcomes: A User's Guide, Third Edition. (Prepared by L\&M Policy Research, LLC, under Contract No. 290-2014-00004-C.) AHRQ Publication No. 17(18)-EHC016-EF. Rockville, MD: Agency for Healthcare Research and Quality; February 2018. www.effectivehealthcare.ahrq.gov. DOI: https://doi.org/10.23970/AHRQREGISTRIESMULTINATL. 


\section{Reviewers (alphabetical):}

Jennifer B. Christian, Pharm.D., M.P.H., Ph.D., FISPE

Vice President, Clinical Evidence

Center for Advanced Evidence Generation

IQVIA

Rachael DiSantostefano, Ph.D., M.S.

Janssen

Nancy A. Dreyer, Ph.D., M.P.H., FISPE

Chief of Scientific Affairs \& Head Center

for Advanced Evidence Generation

IQVIA

Richard E. Gliklich, M.D.

Chief Executive Officer and Chairman

OM1

Louise Morgan, M.S., B.S.N.

American Heart Association 


\section{Contents}

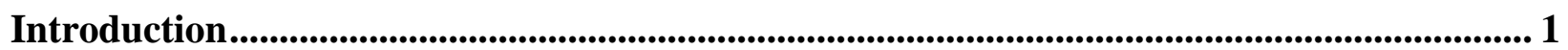

Multinational Registry Models .................................................................................................................... 2

Planning and Design Considerations ....................................................................................................... 3

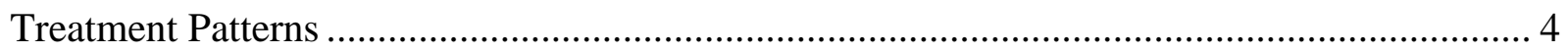

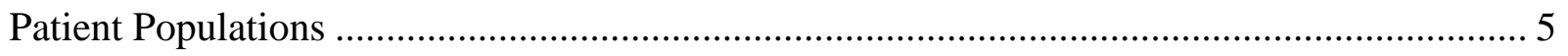

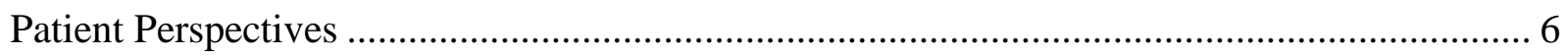

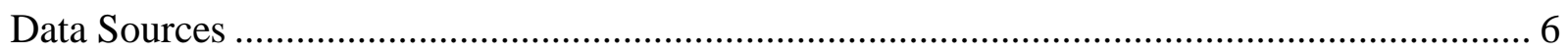

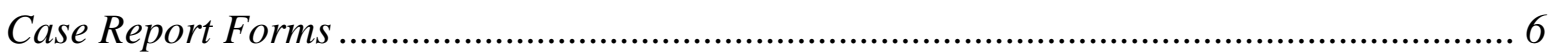

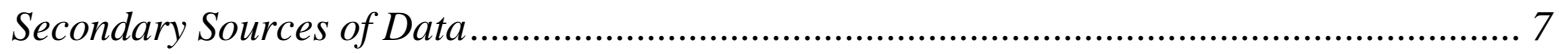

Patient-Reported Outcomes ......................................................................................... 8

Regulatory, Ethical, and Legal Considerations.................................................................. 8

Variations In How Observational Studies Are Defined ....................................................................... 9

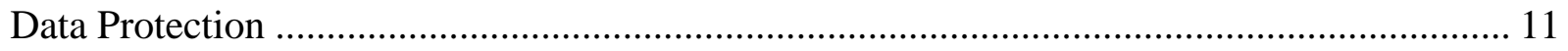

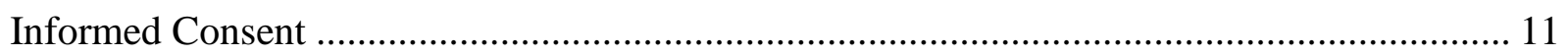

Additional Considerations …………………………….................................................... 12

Regulations and Guidelines Applicable to Observational Studies ............................................ 12

Operational Considerations ................................................................................................................ 13

Recruitment and Retention of Patients and Providers .............................................................. 13

Data Quality Assurance and Data Management ........................................................................ 14

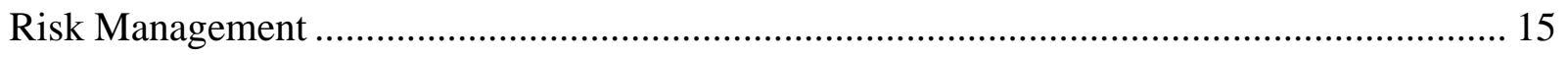

Special Considerations: Emerging Markets.......................................................................................... 15

Conclusion .................................................................................................................................................... 16

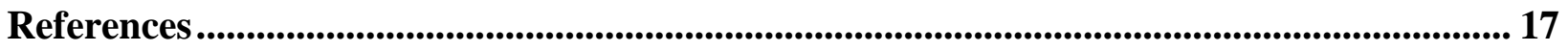


Multinational Registries: Challenges and Opportunities

\section{Figures}

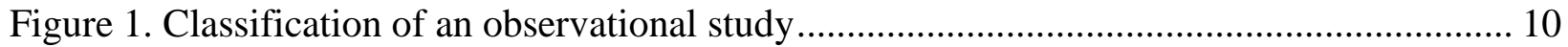




\section{Introduction}

Registries vary widely in geographic scope. Some registries collect data at a local or regional level, while others collect data at a national or multinational level. Multinational registries, also called multicountry, global, or international registries, offer unique research opportunities beyond those offered by national, regional, or local registries. By collecting data from multiple countries, these registries are able to examine geographic variations in disease etiology and progression as well as treatment patterns and clinical effectiveness in various populations. Multinational registries also may be able to enroll larger numbers of patients, which can enhance their capacity to detect adverse events, should they exist, and to better understand rare conditions.

The decision to develop a multinational registry may be driven by many factors. In Europe, for example, registries designed to meet postmarketing requirements for products that are approved through the centralized procedure by the European Medicines Agency (EMA) are nearly always multinational, so that the registry will be able to provide country-specific information in countries where the product will be used. This trend is evidenced in the European Network of Centers for Pharmacoepidemiology and Pharmacovigilance (ENCePP) E-Register of Studies (EU PAS Register), where 123 of the 210 (58.6\%) observational studies listed as requested by a regulatory authority are multinational in scope. ${ }^{1}$

Registry sponsors may also collect data in multiple countries so that a single registry can be used to meet regulatory requirements for several regulatory agencies. Increased collaboration between regulatory agencies (e.g., the EMA, the U.S. Food and Drug Administration [FDA], Health Canada, Japanese Pharmaceuticals and Medical Devices Agency, the Australian Therapeutic Goods Administration) has supported this approach to data collection. ${ }^{2-4}$ Many registries that are designed to meet postmarketing requirements also collect health economic data, with a goal of informing country-specific health technology assessments and reimbursement decisions.

Beyond supporting regulatory or reimbursement decisions, registry sponsors may develop a multinational registry to better understand variations in treatment patterns or outcomes in different populations. For example, the International Registry for Heart and Lung Transplantation (IRHLT) was launched in 1983 to collect longitudinal followup information on transplant recipients. The registry, funded by the International Society for Heart and Lung Transplantation, now captures data on approximately two thirds of all thoracic transplants worldwide, providing a broad picture of practices and outcomes that has been used to inform and change clinical practice. ${ }^{5}$

Collecting data in multiple countries is particularly important in rare disease research, where an expansive approach is often needed to enroll sufficient numbers of patients. An example of a longstanding multinational rare disease registry is the International Collaborative Gaucher Group (ICGG) Registry. ${ }^{6,7}$ The ICGG was designed to examine disease progression and treatment patterns in Gaucher disease, a rare enzyme deficiency that affects fewer than 10,000 patients worldwide. Since its launch in 1991, the registry has collected data from over 6,500 patients in more than 60 countries, resulting in numerous publications and increased knowledge of the natural history of the disease, phenotypic and genotypic variation among patients, diagnosis and treatment patterns, and long-term outcomes. ${ }^{8-10}$ 
Multinational registries can also provide valuable information for more prevalent conditions. Consider GARFIELD-AF (Global Anticoagulant Registry in the FIELD-Atrial Fibrillation), which was designed to evaluate the management and outcomes of patients with newly diagnosed nonvalvular atrial fibrillation (AF) and at least one additional risk factor for stroke. The Registry has already enrolled more than 47,000 patients from 35 countries and is following them for up to six years to assess the global burden of AF and describe "real-life" treatment patterns, making this, the largest active, prospective AF registry in the world. ${ }^{11}$

As highlighted by these examples, multinational registries can provide valuable data to address multiple types of research objectives, but, in order to do so, they must be planned, designed, and operated with several special considerations in mind. While much of the information contained in the document, Registries for Evaluating Patient Outcomes: A User's Guide, ${ }^{12}$ applies to multinational registries, these registries face unique issues resulting from variations in treatment patterns, patient populations, cultural norms, and regulatory and ethical environments. The purpose of this paper is to discuss unique considerations for the planning and conduct of multinational registries, as well as explore the challenges with regards to operational, ethical, and regulatory considerations. Where appropriate, reference is made to other chapters in the User's Guide.

\section{Multinational Registry Models}

As described in Chapter 1 of the User's Guide, ${ }^{12}$ patient registries are classified as product registries, health service registries, and disease or condition registries. These classifications apply to multinational registries as well. Beyond this classification system, it is helpful to distinguish between two models: the central registry model and the registry network model.

In the central registry model, data are collected from investigator sites enrolled in the registry, data are stored in a central database, and the registry is managed by a coordinating center. Data typically are collected in the same manner using the same case report forms (CRFs) at each site. In some cases, CRFs may vary slightly from country to country, for example, to account for differences in standards of care, the availability of certain products, or language translations. Nevertheless, a single protocol governs the study procedures across all sites in all countries. In most cases, registries using this model are sponsored by a single organization or by a consortium of organizations with similar goals. Examples of registries utilizing the central registry model include the ICGG Gaucher Registry, ${ }^{6-10}$ the Cochlear Pediatric Implanted Recipient Observational Study (Cochlear P-IROS), ${ }^{13}$ the Gulf Locals with Acute Coronary Syndrome Events Registry (Gulf COAST Registry), ${ }^{14}$ GARFIELD-AF, ${ }^{15}$ and many others.

In contrast, data in the registry network model are collected and aggregated at the network level from individual registries that are operating at the national or regional level. Each individual registry has its own protocol, investigator sites, CRFs, and database. Typically, the individual registries share a common data model for some of the data elements, and those data elements are extracted and aggregated at the network level. Common data management and data quality standards are necessary to ensure that all data in the network database are of sufficient quality to support the intended analyses. 
An example of a registry that uses the network model is Psonet, an international network of population-based registries designed to monitor the long-term effectiveness and safety of systemic agents used in treatment of psoriasis. ${ }^{16}$ At the time Psonet was designed, multiple national-level registries existed to track outcomes for psoriasis patients treated with systemic agents; however, these registries typically had small patient populations and limited geographic coverage. To improve surveillance, the Psonet investigators created a network of nine European registries and one Australasian registry. The registries agreed on the Psonet protocol and a common set of variables and procedures; recent work has focused on understanding heterogeneity in patient populations. ${ }^{17}$ The IRHLT (described previously) is another example of a registry that uses the network model. This registry incorporates data from 16 national-level transplantation registries as well as data submitted directly from 80 institutions. In this case, the network model is efficient, as many countries already collect the necessary data in national registries. ${ }^{5}$ There are many other examples where multiple registries have been combined into one network model for the purpose of evaluating a condition, intervention, or treatment in a broader population, including multinational studies of ceramic-on-ceramic hip implants ${ }^{18}$ and colonic stenting for invasive bowel obstructions. ${ }^{19}$

Both the central registry and the registry network models have strengths and limitations. The central registry model offers the sponsor(s) full control over the design of the study, the data collected, and the analyses. These registries, however, are time consuming and resource intensive to establish and conduct, particularly if large numbers of patients or long-term followup data are needed. In addition, in cases where the patient population is limited (e.g., rare diseases), a new central registry may face enrollment challenges if most patients and sites are already participating in existing registries. In comparison, sponsors of a registry network have limited control over the data collected in the participating registries and must work as a collaborative team with those registries. In cases where registries have already been developed and are collecting data, it may be challenging to make changes to the established data collection procedures. Differences in coding and data ownership arrangements may also make sharing data more challenging. In addition, patient informed consent must allow for the data to be shared within the registry network. However, the network model may be more efficient in terms of both costs and time in cases where there are existing registries of high quality already established at the individual country or region level.

Before developing a new multinational registry, it is useful to consider the feasibility of each model, as well as the trade-offs in terms of sponsor control and efficiency. The following sections discuss considerations related to the next steps of planning, designing, and operating multinational registries. Many of these considerations apply to both the central registry and the registry network models; however, registries using the network model face some additional challenges related to governance, data harmonization, data sharing, and change management that are beyond the scope of this paper.

\section{Planning and Design Considerations}

The planning and design of multinational registries follows the main steps outlined in the User's Guide, with some notable additions. In particular, consideration of the potential differences between the countries represented in the registry is critical, as these differences may have a substantial impact on the study design as well as the feasibility, timelines, and cost of the study. 
Significant areas of variation, including treatment patterns, patient populations, patient perspectives and data sources, are described below, together with strategies for addressing the variations.

\section{Treatment Patterns}

Treatment patterns often vary across geographic regions due to multiple factors, including differences in approved indications, coverage decisions, and clinical guidelines. Products may be approved for different indications in different countries or regions, which can lead to the use of the product by patients with different characteristics, including varying levels of severity of conditions in each country or region. This, in turn, may affect the perceived effectiveness or safety of the product if the registry does not take into account these differences during the collection, analysis, and interpretation of the data. For example, natalizumab is approved in the European Union (EU) for patients who have failed two or more therapies for relapsing-remitting multiple sclerosis, while, in the United States, the therapy is used more widely. ${ }^{20,21}$ Differences in indication and use may help to explain the geographic variability in the incidence rates of Progressive Multifocal Leukoencephalopathy (PML), a serious adverse event related to treatment with natalizumab. ${ }^{22}$

Differences in health insurance coverage decisions may affect treatment patterns in a similar manner. Access and reimbursement levels may differ among countries, which can impact providers' and patients' ability and willingness to use a specific product. For example, when studying generic medications, it is difficult to predict and monitor changes in pharmacies' purchasing patterns, which becomes particularly challenging when investigating products such as biosimilars, which are administered through infusion and not dispensed through regular pharmacy outlets. It can also be challenging to differentiate drugs available by prescription with those available without a prescription. In a recent study of medication use during pregnancy in four EU countries, it was difficult to compare prescription drug use across countries since some medications not usually covered by health insurance are reimbursed during pregnancy; practices varied by country and time period. ${ }^{23}$ Also, in some situations, coverage decisions may limit access of the treatment or procedure to some groups of patients in one country, whereas in other countries that treatment or procedure may be more widely available. Similar to the example with approved indications, regional differences can lead to product use for diverse conditions across countries, which must be taken into account during data analysis and interpretation.

Beyond regulatory and coverage decisions, treatment patterns may vary across geographic regions for other reasons. A recent comparison of treatment strategies for older breast cancer patients in the Netherlands and Ireland, for example, found that treatment differed significantly on all treatment modalities (guideline-adherent locoregional treatment, endocrine therapy, and chemotherapy), with more locoregional therapy provided in the Netherlands and systemic therapy provided in Ireland. ${ }^{24}$ The authors suggested that the differences resulted in part from discrepancies in the guideline recommendations between the two countries, as well as an increased likelihood to deviate from the guidelines in the Netherlands.

The use of different clinical guidelines can have a substantial impact on treatment patterns. The American Gastroenterological Association, for example, recommends annual or biannual colonoscopic surveillance for neoplasia in patients with inflammatory bowel disease-related 
colitis, depending on whether patients are considered high risk or average risk. ${ }^{25}$ In contrast, the British Society of Gastroenterology recommends colonoscopic surveillance on a 1-year, 3-year, or 5 -year basis, depending on risk assessment. ${ }^{25}$

Differences in the practice of medicine can be difficult to identify. Multinational registries can benefit from engaging with key opinion leaders from each country represented in the registry to identify and mitigate the potential impact of these types of variations. The registry also may need to collect additional information, such as the reason for using a particular product (if indications vary), information on disease severity, or other covariates that may affect assessments of effectiveness or safety. Feasibility assessments related to enrollment goals and pilot testing of the CRFs may also be useful to identify issues before launch of the full registry.

Lastly, it is important to note that treatment patterns and standard of care may change during the lifespan of the registry. A new indication for a product or a new product may be approved, coverage decisions may be revised, or clinical guidelines may be updated. These types of changes can have an effect on the use of a product in a single country or in several countries, which may in turn impact patient enrollment and followup and, in some cases, necessitate changes to the inclusion and exclusion criteria of the registry or other modifications to the protocol. Thus, multinational registries must assess the potential for treatment pattern variation at the outset and plan for ongoing monitoring of factors that may influence treatment patterns throughout the life of the registry.

\section{Patient Populations}

Beyond variations in treatment patterns, other factors can result in differences among the eligible or enrolled patient populations in different countries. Some countries have younger and rapidly growing populations, as compared to other countries where the population is aging or growth is stagnant. As a result, incidence and prevalence of diseases may vary widely across regions, affecting the number of eligible patients for a potential registry. In Europe, geographical differences in cancer incidence and prevalence have been documented across countries. Indeed, a study of cancer epidemiology in eight Southern and Eastern European countries found large variations in incidence rates per 100,000 population in men of prostate cancer (16.8 in Romania vs. 59.5 in the Czech Republic), stomach cancer (11.2 in the Czech Republic vs. 20.5 in Hungary), and lung cancer (50.0 in Romania vs. 94.6 in Hungary). ${ }^{26}$ Variability in cancer incidence and mortality have also been documented on a global scale by the International Agency for Research on Cancer (IARC). IARC has described wide geographic differences in incidence and mortality rates for specific types of cancer as well as for all cancers. ${ }^{27,28}$

When planning a registry, developing an understanding of the available patient populations in each country included within the registry is an important step that may influence the decision to focus on specific countries/regions where large populations may be available for recruitment. An assessment of the number of patients who would potentially meet the enrollment criteria for the registry should be conducted while designing the study. Differences in diagnostic criteria and/or screening rates across countries can result in significant geographic and clinical variability in registry patient populations. For example, countries that do not implement standard breast or prostate cancer screening procedures often diagnose these cancers at later stages leading to significant differences in survival odds, patient signs and symptoms, and burden of illness (e.g., 
advanced stage prostate cancer often manifests with bone metastases, which are extremely painful and require expensive surgical treatment).

As discussed in later sections, inclusion of additional countries requires greater resources to support translations, ethics committees' approvals, and site recruitment and management. Therefore, careful feasibility assessments should be conducted to support the rationale for selecting and expanding to additional countries. Rare disease registries are an exception to this principle, in that they seek to enroll as many patients as possible from a very limited global pool of patients; given the extremely small size of the population, these registries often enroll patients from any country.

\section{Patient Perspectives}

In recent years, significant attention has been paid to increasing patient-centeredness in clinical research. Efforts to increase patient-centeredness often focus on incorporating the patient perspective in the design and conduct of clinical research studies, such as patient registries. Patient perspectives may shape the research questions that are addressed by a patient registry (such as focusing on outcomes of interest to the patient or comparing treatment modalities). Patient feedback may also inform the design of the study, including the schedule of followup visits and the selection, mode of administration, and timing of patient-reported outcome (PRO) measures, as well as recruitment and retention efforts. Gathering and incorporating patient perspectives into a registry introduces many challenges that are beyond the scope of this document; however, it should be noted that multinational registries may face the additional challenge of different patient perspectives and priorities in different countries and regions. For example, patients in one country may express strong preferences related to research priorities or the study design that are not compatible with the preferences expressed by patients in other countries. Before embarking on a plan to gather patient perspectives, registry sponsors should have a clear plan for addressing different preferences among different patient groups.

\section{Data Sources}

As observational studies, patient registries typically rely on data that are collected as part of routine patient care. However, the data that are recorded during clinical care may vary by country, either because of differences in how and what data are documented or because of differences in the frequency and types of assessments or tests that are performed. The sources of data that may be used to populate the registry include CRFs, secondary data sources, and PRO measures.

\section{Case Report Forms}

CRFs are used to systematically collect information from health care providers, either on paper or in electronic format. When collecting data in more than one language and/or culture, appropriate translation and linguistic validation of CRFs is critically important to maintain a high quality of systematic data collection. One of the first steps is for the CRF to be translated into the common language(s) of each study region. In keeping with International Society For Pharmacoeconomics and Outcomes Research (ISPOR)'s Principles of Good Practice for translation, this process requires preparation, multiple forward translations, reconciliation of 
these forward translations, back translations, review of the back-translated text, and harmonization of multiple back translations to ensure consistency. ${ }^{29}$

The translated CRFs also should be linguistically valid in each study region. This does not always naturally follow from the rigorous translation process. For example, though persons in the United States and the United Kingdom (UK) both commonly speak English, content validity of the same English translation may differ between the two nations due to different cognitive interpretations. For example, consider patient-reported weight; a patient in the United States would typically write the full amount in pounds, while a patient in the UK would typically write the amount in stone or pounds or possibly kilograms. To ensure linguistic validation, each translated CRF also should undergo cognitive debriefing, where it is tested on a small group of patients or lay people from the area of study to check understandability, interpretation, and cultural relevance. The results of the cognitive debriefing interviews are then compared against one another and against the original translation. Any discrepancies between the original translation and the feedback from native speakers are corrected during a proofreading step. ${ }^{29}$

Additionally, CRFs implemented in multiple countries must allow for variability in standard of care. Because registries are observational, additional diagnostic or monitoring procedures such as laboratory tests are not undertaken unless they are within the scope of normal practice. Combined with differences in national guidelines, policies, and regulations (e.g., recommended screening procedures, approved medications, etc.), this makes variation in data availability commonplace for multinational registries. For this reason, CRFs must be tailored appropriately for each country.

\section{Secondary Sources of Data}

Some registries incorporate information from other sources, such as administrative claims data or electronic health record (EHR) data. While the challenges of linking a registry to these other sources of data are well-documented (see Chapters 6 and 15-18 of the User's Guide ${ }^{12}$ ), once accomplished, linkage may reduce the data entry burden on sites and/or provide information that is otherwise unavailable in the registry (such as capturing medication adherence rates, additional comorbidities, prior clinical history not captured within the CRF, and long-term followup.

For multinational registries, linkage to secondary data sources is challenging due to inconsistent availability and requirements for access to source data. Rather, administrative healthcare claims data and/or EHR data are available in certain countries, and, within these countries, the types of available data may be restricted to regional or localities, and can be difficult to link with individual patients. A coordinated effort is needed to extract a common set of core data elements from each source of information, accounting for variations in data structure, coding conventions, format, language differences, standards of care, and clinical care. In most cases, only a subset of participants within a multinational registry will have administrative or healthcare data to link; therefore, the analyses would be limited to a subgroup of the entire study population.

Given these challenges, use of secondary data sources in a multinational registry may be used for sub-studies nested within the registry; for example, a multinational registry examining effectiveness of a new medication may include secondary data from one country to examine specific questions related to cost-effectiveness even when such data are not available in other 
countries. This strategy is particularly relevant in cases where a registry is designed to meet multiple regulatory or reimbursement requirements in different countries.

\section{Patient-Reported Outcomes}

As discussed in Chapter 5 of the User's Guide, PROs are defined as "a measurement based on a report that comes directly from the patient (i.e., the study subject) about the status of a patient's health condition, without amendment or interpretation of the patient's response by a clinician or anyone else."12

Collection of PROs in multinational registries requires careful planning. PRO instruments may not be available in all necessary languages. For example, an available translation may not be linguistically validated in a specific country. In cases where the PRO instrument has not been linguistically validated, registry developers may consider validating the instrument within the registry, as the study is running, or not collecting responses to that PRO instrument in the particular country. In either case, the scientific impact on the study must be considered as part of the decision-making process.

Once the PRO instrument has been selected, the frequency of assessments should be determined. The assessment schedule must be consistent with the study aims, length of recall for the response options, the disease(s)/condition(s) or treatment(s) of study, and the planned analyses. In addition, multinational registries must take into account the potential for variation in routine clinical care and followup visit schedules. For example, patients with a chronic condition may be seen every three months in some countries, but only every six months in other countries. Registries that propose to collect PROs at routine physician office visits will need to plan for these types of variations; alternatively, registries may elect to collect PROs directly from patients at regularly scheduled intervals to address this issue. The frequency of data collection from patients needs to be managed as thoughtfully as that from clinicians, recognizing that patients rarely receive any reimbursement, and no matter how altruistic they may be, patients will experience reporting fatigue, will provide inconsistent followup and may drop out completely before the study is finished. Differences as these should be considered in the planning and design phases, as well as in the statistical analysis plans for the registry.

\section{Regulatory, Ethical, and Legal Considerations}

In addition to the planning and design considerations discussed above, assessment of the regulatory, ethical, and legal environments in which a multinational study will operate is a critical step in developing the registry. The regulatory framework for observational studies differs among countries, as do regulations governing informed consent and data protection; these differences necessitate careful planning to avoid unanticipated delays and/or changes to the registry design. The purpose of the following sections is solely to provide information that will help readers understand the issues relevant to conducting multinational registries; this section is not intended to provide specific legal opinions or regulatory advice. Legal advisors should always be consulted early in the planning process to address specific issues and to ensure that all applicable rules and regulations are followed. 


\section{Variations In How Observational Studies Are Defined}

Registries are, by definition, observational in nature. However, the definition of an observational study and the regulatory framework governing the conduct of such studies vary across countries and regions. In fact, even the terminology for an "observational study" is not harmonized at a global level. The terms "phase IV," "noninterventional," prospective observational study," "postmarketing study," and "postauthorization safety study" are all used to refer to observational studies, depending on the study design and the country of interest. The determination of whether a multinational registry will be considered an observational study in the countries of interest depends largely on the registry design and purpose, as well as whether the registry examines use of a specific product(s). An assessment of whether the registry is likely to be viewed as observational is an important step in planning the registry, and one that should be undertaken early in the planning process.

In the US, the term "observational study" is used to refer to the type of research conducted by patient registries. The observational studies conducted through patient registries typically are considered "research involving human subjects," as defined by the Federal Policy for the Protection of Human Subjects, or the "Common Rule." As such, these observational studies are governed by Institutional Review Board (IRB) guidelines. The FDA can also require the conduct of observational studies at the time of marketing authorization in the form of a postmarketing commitment or postmarketing requirement. Chapter 7 of the User's Guide provides an in-depth discussion of the ethical, legal, and regulatory principles applicable to the conduct of patient registries within the US.

In the EU, the term "noninterventional study" is used currently to refer to observational study designs. ${ }^{1}$ The EMA defines a "non-interventional trial/study" as "a study where the medicinal product(s) is (are) prescribed in the usual manner in accordance with the terms of the marketing authorization. The assignment of the patient to a particular therapeutic strategy is not decided in advance by a trial protocol but falls within current practice, and the prescription of the medicine is clearly separated from the decision to include the patient in the study. No additional diagnostic or monitoring procedures shall be applied to the patients and epidemiological methods shall be used for the analysis of collected data." ${ }^{30}$

Other countries use the term "Phase IV" to refer to observational study designs. For example, in Canada, "Phase IV" refers to any postmarketing study of products that have received marketing authorization and are being used according to marketing authorization. "Phase IV" is also commonly used in India and China.

The use of different terms to refer to observational studies reflects a deeper discrepancy in what is considered observational versus interventional research, a distinction that affects the regulatory requirements that apply to a study. At a high level, a study is considered to be observational or noninterventional if it meets the following criteria: 1 ) it does not involve a specific medicinal product OR it involves a medicinal product that has received marketing authorization; 2) the product will be used in accordance with the marketing authorization; and 3) the study will be conducted per standard of care. This classification is presented in Figure 1. 
Figure 1. Classification of an observational study

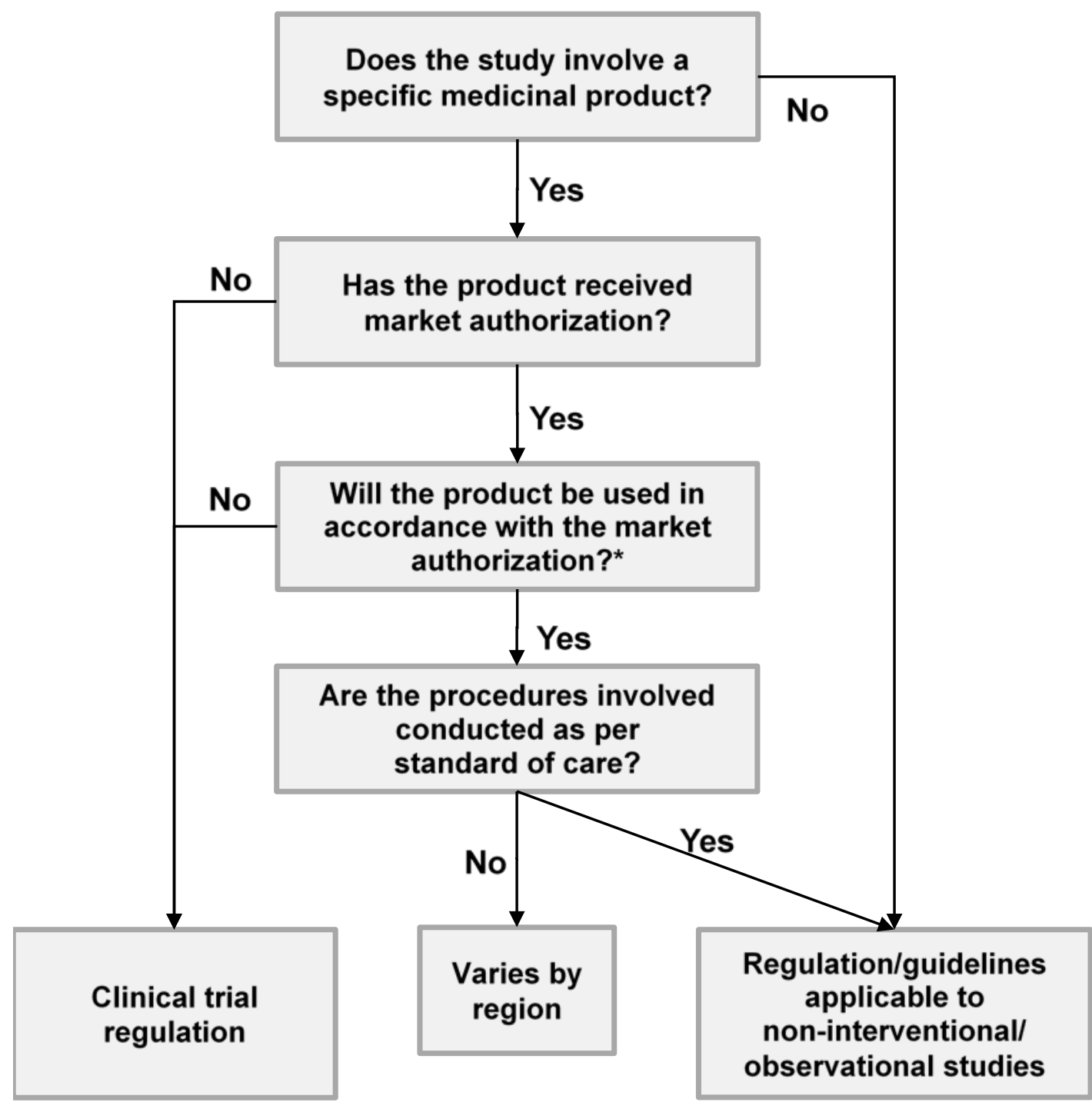

*Note that off-label use can be studied outside of the Clinical Trial Regulations in some countries, e.g., the United States, where access is not restricted to approved indications, providing that there are no inducements for off-label prescription.

An area where the classification of a study as observational becomes more complicated is in the interpretation of whether the procedures involved in the study are considered routine or standard of care. In some countries, a study may be considered observational even if some of the additional monitoring or other procedures are not part of routine care; the determination depends on the types of additional monitoring or other procedures that are required by the study. For example, in some countries, a study that includes additional monitoring in the form of collection 
of PRO data that are not routinely collected as part of standard of care may still be considered observational.

Within the EU, the distinction between noninterventional and interventional can be particularly challenging, as both the interpretation of the terms and the standard of care may vary across countries. As a result, a study that is considered noninterventional in one country may be considered interventional in another, or studies that are considered observational in the United States may be considered interventional in European countries. Because the designation of a study as observational or interventional determines the applicable regulatory requirements and guidelines (e.g., in the EU, interventional studies require a full clinical trial submission and compliance with clinical trial regulations), it is important to understand early in the planning process how a proposed registry design will be perceived in each of the countries of interest.

\section{Data Protection}

As with the concepts of observational versus interventional, the concepts of privacy and data protection differ across countries. In the EU, health-related data is protected under laws governing the collection, processing, and movement of personal data. ${ }^{31}$ Registries that collect personal data in the EU must disclose to participants in the informed consent form 1) the purpose of the data collection and processing; 2) all recipients of the data; 3) the participant's rights; and 4) any plans to transfer data outside of the EU to a country that does not have an adequate level of data protection. Data protection requirements are enforced by the National Data Protection Authority, and approval may be required for the conduct of clinical studies, depending on local regulations.

In comparison, in the United States, health information is protected under the Health Insurance Portability and Accountability Act (HIPAA). ${ }^{32}$ HIPAA describes three types of health information: individually-identifiable health information, limited data sets, and de-identified data; the type of health information that is being collected by the registry determines the requirements for informed consent. Informed consent forms must disclose to participants 1) the purpose of the data collection; and 2) the participant's rights. Chapter 7 of the User's Guide ${ }^{12}$ provides an in-depth discussion of HIPAA and its applicability to patient registry research.

As this comparison illustrates, the requirements for data protection differ across countries and may influence the type of data collected (e.g., contact information for followup visits) as well as plans to transfer data collected in one country to another country for analysis.

\section{Informed Consent}

Data protection requirements also affect the informed consent process for multinational registries. Because observational studies do not alter the care that a patient would receive in routine clinical practice, the informed consent documents for an observational study typically do not focus on potential risks of treatment, as is common with clinical trial informed consents. Instead, the informed consent for an observational study focuses on the purpose of collecting the data, how the data will be handled, and who is going to see the data - in other words, data protection. As a general principle, informed consent is required for access to and the use of any identifiable or potentially identifiable health data that will be collected in a registry. However, there is no consistent guidance at an international level for when and how to obtain this consent. 
For example, in the US, an IRB will typically waive the requirement for written informed consent for studies that rely on de-identified, retrospective data. However, data that are considered de-identified in the United States under HIPAA may not be considered anonymous in the EU under the Data Protection Act. Further, requirements for how informed consent are collected (e.g., electronic consent) also varies between countries. ${ }^{1}$

Once it is determined that consent is required, development of the informed consent forms can also introduce challenges particularly when pediatric populations are involved. For example, the study may need consent for adults, assent for pediatrics with two to three variations on the form depending on age of child, and consent for the parent or guardian of a pediatric patient. Other studies could include consent from a caregiver, consent from a pregnant woman, and consent from the partner of a pregnant woman. Furthermore, each consent form may need to be adapted to local country's requirements and presented in multiple languages to accommodate diverse patient populations.

\section{Additional Considerations}

Beyond data protection and informed consent, other differences across countries in the regulations and guidelines governing observational studies may influence the design and implementation of a multinational patient registry. For example, in some countries such as the US, the approval of observational studies is largely driven by IRBs or ethics committees, whereas in other countries, data privacy authorities or health authorities may also need to weigh in. These differences may result in preparation of multiple sets of approval documents and well as very different timeframes for approval (e.g., a few weeks vs. several months). ${ }^{33}$ Requirements for safety reporting also vary by country and region and by study design and sponsor. As a result of these differences, multinational registries often must develop detailed protocols outlining appropriate procedures in each country of interest.

\section{Regulations and Guidelines Applicable to Observational Studies}

As is evident from the preceding discussions, the regulations and guidelines that are applicable to observational studies are not harmonized globally. To ensure compliance, registry sponsors must review and understand the applicable regulations and guidelines for each country in which the registry will be implemented. A full review of the applicable regulations and guidelines for all countries in which registries may be implemented is beyond the scope of this document. There are, however, some guidelines that are relevant to patient registries and applicable at an international level, which are noted here:

- International Society for Pharmacoepidemiology (ISPE) Guidelines for Good Pharmacoepidemiology Practices (GPP) ${ }^{34}$

- Declaration of Helsinki ${ }^{35}$

- International Conference on Harmonisation (ICH) E6 Good Clinical Practices $(\mathrm{GCP})^{36}$

- ISPE Data Privacy, Medical Record Confidentiality, and Research in the Interest of Public Health ${ }^{37}$ 
Lastly, it should also be noted that the regulations and guidelines governing the conduct of observational studies are a dynamic area, and frequent change should be anticipated.

\section{Operational Considerations}

Multinational registries face many of the same challenges as other types of registries in the implementation and operational phases; these challenges are discussed in detail in Chapters 1014 on “Operating Registries” in the User's Guide. ${ }^{12}$ The following section highlights challenges related to recruitment and retention, data collection and quality assurance, and risk management that are unique to multinational registries.

\section{Recruitment and Retention of Patients and Providers}

As discussed in previous sections, recruitment plans for multinational registries must take into account variations across countries in disease epidemiology, treatment patterns, and access to treatment as determined by regulatory and reimbursement decisions (e.g., approved indications, restricted access programs, requirement for prior authorizations, etc.). Beyond these considerations, recruitment efforts must be tailored to each of the countries in which patients will be enrolled. This includes developing recruitment materials that comply with local regulations, are culturally appropriate, and are translated into the relevant languages for the populations of interest. In particular, it is essential to ensure that site contract templates are appropriate for each country of interest and address the necessary legal requirements. Registries may also elect to partner with organizations in different countries to facilitate recruitment; for example, nationallevel professional organizations or patient advocacy organizations can help to share information about a registry in support of recruitment efforts.

Sometimes sites are chosen to represent the national standard of care mix. This generally will result in the inclusion of inexperienced investigators, which poses an entirely different burden on registry conduct than would a study that relies substantially on experienced investigators with established infrastructures for clinical trials and other types of research. GARFIELD-AF addressed this by creating a training portal, with robust and refreshed training materials about the protocol, data collection tools and other study processes. Sites received training and periodic retraining as new or replacement staff rotated onto the project. Equally important, substantial attention is devoted to keeping sites interested and involved, since many sites have been participating for several years. Motivation is provided through local workshops and investigator meetings that allow for registry groups to occur throughout the registry life cycle. Investigators receive information on an on-going basis that compares global outcome data on treatment patterns and outcomes with that in their own countries.

Another feature of GARFIELD-AF that was intended to enhance retention is the use of a global advisory board to guide study design and execution. A national share-back program was established where national coordinating investigators or country leads for the registry drive country-specific strategies including site recruitment, local meetings and publications. Central analytic support is used to support content development. Channels were created for investigators to log their research ideas through date and time-stamping suggestions made to the Steering Committee. The Steering Committee and National Coordinating Council meet annually to 
exchange experience, share key learnings and review data. These processes have resulted in a productive research program. $^{38}$

Once patients have been enrolled into the registry, the focus shifts to retaining those patients for the duration of the followup period, which may extend for several years. Again, understanding local regulations and the potential impact on retention efforts is critical; for example, within the US, contract research organizations (CROs) may collect patient contact information, such as the patient's name, phone number, email address, mailing address, and use this information to contact patients directly to obtain followup information. The contact may take the form of sending links via email to complete web-based surveys or sending and receiving forms by mail. In other countries, CROs are not permitted to contact patients directly to obtain followup information; instead, this activity must be completed by the patient's physician (or physician office staff). The ability to obtain followup data via linkage with other data sources (e.g., national death indices, administrative data) also varies by country and region. Linkage with the National Death Index in the United States is a feasible strategy for obtaining mortality data for some registries, but linkage with death indices in the EU is much more challenging and often not feasible due to data protection restrictions.

One area of particular complexity that affects both recruitment and retention is the provision of incentives to encourage patient participation. Views on incentivizing patients differ across countries and regions; in the US, for example, sometimes patients are provided with a nominal incentive, commensurate with the amount of time spent completing study-related activities. These incentives may take the form of small payments (often in the form of gift cards); in addition, some registries provide patients with newsletters or other educational materials as part of participation. Within the EU, rules governing patient incentives are far more restrictive. Nonetheless, patients report that even modest compensation is important to them since it acknowledges the value of their contributions. ${ }^{39}$

As a result of these variations, registries operating in multiple countries typically need a defined recruitment and retention plan for each country in which the registry will enroll patients.

\section{Data Quality Assurance and Data Management}

Consideration must be given to the completeness, consistency and accuracy of the data across countries. At the outset, any registry materials - such as CRFs, data definitions, and training manuals - that are translated into other languages should undergo strict quality assurance measures to ensure that the terms are translated properly (e.g., back translation). The ability to provide training and ongoing support to sites in the appropriate languages is also important.

GARFIELD-AF uses a process of regular review of site data to gauge quality. The frequency of remote site contact is then adjusted based on recruitment performance and data quality. Remote site contact calls are tailored to ensure that inexperienced sites get more help than sites that are more experienced and need less active contact. As with any relevant registry, changes are made to the CRFs as science evolves over time, and the data management plans are adjusted accordingly. 


\section{Risk Management}

In managing a multinational registry, the real-world dynamics of the study usually pose the biggest challenge. For example, in a drug exposure registry in which the drug is new to market, patient enrollment into the registry is dependent upon drug access and reimbursement as well as market uptake of the drug. The five-year enrollment plan for such a registry could be derailed by delays in reimbursement or market uptake, perhaps due to the drug being classified as a secondor third-line therapy and/or requiring prior authorization to prescribe and dispense. For a given drug, any or all of these factors could vary by country. The enrollment schedule may also be derailed by delays in obtaining ethics or regulatory approval in some countries, or by the launch of new studies competing for the same patient population. Beyond enrollment, changes in the regulatory environment or in standards of care or treatment patterns (e.g., the introduction of a new therapy, introduction of new indication for existing therapy) may necessitate changes in the registry.

Given the complexities of operating a multinational registry, operational risk management is necessary throughout the life cycle of the registry. Potential risks should be identified in advance, on a country-by-country basis, and risk management activities should be dynamic and ongoing, making use of study feasibility information and the most up-to-date enrollment and patient retention metrics.

\section{Special Considerations: Emerging Markets}

Some multinational registries focus on emerging markets, such as China, India, Brazil, Indonesia, and Turkey, where medical spending is rapidly increasing. As these countries become larger consumers of medical products, it is important for registries to enroll patients in these regions to obtain a representative global sample - or, in some cases, to increase the number of patients eligible for enrollment (e.g., in rare disease research).

The considerations outlined in previous sections, particularly those related to variations in patient population and changes in standard of care, apply here and may in fact be even more important when including emerging markets in a multinational registry. In addition, the following factors should be considered:

- The regulatory environment may be changing rapidly in these countries and must be monitored closely during planning and operational phases of the study.

- The regulatory requirements and approving bodies may also be less clearly defined than in some areas with well-established history of conducting observational research. Observational and interventional studies may be reviewed by the same bodies and held to the same standards.

- Many sites may be research-naïve and will need training to achieve consistent and high quality data collection. For example, an awareness program and educational materials may be needed to overcome a lack of familiarity with observational research. 
- Site compensation may be challenging if the country does not have guidelines as to what constitutes fair-market compensation for site participation.

- Limited availability of ethics committee may introduce delays.

Paper CRFs may be necessary in some cases due to lack of access to technology and reliable Internet service.

\section{Conclusion}

Multinational registries offer great potential to address many types of research questions. These registries may also provide an efficient infrastructure to meet various regulatory and reimbursement requirements related to development of a new therapy. Yet, multinational registries face many challenges because of the variation across countries in terms of patient demographics, how and where clinical care is provided, differences in access to treatment and treatment patterns, and ethical, regulatory, and legal environments. While some of these factors, like variations in patient population and standard of care, are unlikely to change, increased harmonization of ethical and regulatory requirements would support the efficient use of multinational registries to address a wide range of research questions. At present, sound planning and design, along with proactive risk management, are essential for developing and implementing a successful multinational registry. 


\section{References}

1. ENCePP: European Network of Centres for Pharmacoepidemiology and Pharmacovigilance: Study Search. http://www.encepp.eu/encepp/studySearch.h tm. Accessed January 10, 2017.

2. Eriksson S, Helgesson G. Potential harms, anonymization, and the right to withdraw consent to biobank research. Eur J Hum Genet. 2005 Sep;13(9):1071-6. PMID: 15986039.

3. Dal Pan G, Arlett P. The US Food and Drug Administration-European Medicines Agency Collaboration in Pharmacovigilance: Common Objectives and Common Challenges. Drug Safety. 2015 January 1;38(1):13-5.

4. Elger B, Biller-Andorno N, Mauron A, et al. Consent and Use of Samples. Ethical Issues in Governing Biobanks: Global

Perspectives. Burlington, VT: Ashgate Pub. Ltd.; 2008. pp. 57-88.

5. Stehlik J, Edwards LB, Rowe A, et al. ISHLT International Registry for Heart and Lung Transplantation - three decades of scientific contributions. Transplant Rev (Orlando). 2013 Apr;27(2):38-42. PMID: 23465193. Epub March 8.

6. ICGG Gaucher Registry. https://www.registrynxt.com/Gaucher/Pages /Home.aspx. Accessed January 10, 2017.

7. ICGG Gaucher Registry. ClinicalTrials.gov. NCT00358943.

https://clinicaltrials.gov/ct2/results?term=N CT00358943. Accessed January 10, 2017.

8. Khan A, Hangartner T, Weinreb NJ, et al. Risk factors for fractures and avascular osteonecrosis in type 1 Gaucher disease: a study from the International Collaborative Gaucher Group (ICGG) Gaucher Registry. J Bone Miner Res. 2012 Aug;27(8):1839-48. PMID: 22692814. Epub June 14.
9. Rosenbloom B, Balwani M, Bronstein JM, et al. The incidence of Parkinsonism in patients with type 1 Gaucher disease: data from the ICGG Gaucher Registry. Blood Cells Mol Dis. 2011 Jan 15;46(1):95-102. PMID: 21067946. Epub Nov 12, 2010.

10. Weinreb NJ, Aggio MC, Andersson HC, et al. Gaucher disease type 1: revised recommendations on evaluations and monitoring for adult patients. Semin Hematol. 2004 Oct;41(4 Suppl 5):15-22. PMID: 15468046 . Epub Oct 7.

11. Kakkar AK, Mueller I, Bassand JP, et al. International longitudinal registry of patients with atrial fibrillation at risk of stroke: Global Anticoagulant Registry in the FIELD (GARFIELD). Am Heart J. 2012;163(1):139.e1.

12. Gliklich R, Dreyer N, Leavy M, eds. Registries for Evaluating Patient Outcomes: A User's Guide. Third edition. Two volumes. (Prepared by the Outcome DEcIDE Center [Outcome Sciences, Inc., a Quintiles company] under Contract No. 290 200500351 TO7.) AHRQ Publication No. 13(14)-EHC111. Rockville, MD: Agency for Healthcare Research and Quality. April 2014.

http://www.effectivehealthcare.ahrq.gov/reg istries-guide-3.cfm. Accessed January 10, 2017.

13. Sanderson G, Ariyaratne TV, Wyss J, et al. A global patient outcomes registry: Cochlear paediatric implanted recipient observational study (Cochlear P-IROS). BMC Ear Nose Throat Disord. 2014;14:10. PMID: 25317075. PMCID: 4196206.

14. Zubaid M, Thani KB, Rashed W, et al. Design and Rationale of Gulf locals with Acute Coronary Syndrome Events (Gulf Coast) Registry. The open cardiovascular medicine journal. 2014;8:88. 
15. Kakkar AK, Mueller I, Bassand JP, et al. Risk profiles and antithrombotic treatment of patients newly diagnosed with atrial fibrillation at risk of stroke: perspectives from the international, observational, prospective GARFIELD Registry. PLoS One. 2013;8(5):e63479.

16. Lecluse LL, Naldi L, Stern RS, et al. National registries of systemic treatment for psoriasis and the European 'Psonet' initiative. Dermatology. 2009;218(4):34756. PMID: 19077384. Epub Dec 17, 2008.

17. Ormerod AD, Augustin M, Baker C, et al. Challenges for synthesising data in a network of registries for systemic psoriasis therapies. Dermatology. 2012;224(3):23643. PMID: 22678413. Epub June 9.

18. Sedrakyan A, Graves S, Bordini B, et al. Comparative effectiveness of ceramic-onceramic implants in stemmed hip replacement: a multinational study of six national and regional registries. J Bone Joint Surg Am. 2014 Dec 17;96 Suppl 1:34-41. PMID: 25520417. Epub Dec 19.

19. Jimenez-Perez J, Casellas J, Garcia-Cano J, et al. Colonic stenting as a bridge to surgery in malignant large-bowel obstruction: a report from two large multinational registries. Am J Gastroenterol. 2011 Dec;106(12):2174-80. PMID: 22085816. Epub 2011/11/17. eng.

20. Medication Guide: Tysabri. http://www.fda.gov/downloads/Drugs/Drug Safety/ucm089809.pdf. Accessed January 10, 2017.

21. Tysabri: EPAR - Summary for the public. http://www.ema.europa.eu/docs/en_GB/doc ument_library/EPAR_-

_Summary_for_the_public/human/000603/ WC500044688.pdf. Accessed January 10, 2017.

22. Transatlantic workshop on Drug-related Progressive Multifocal Leukoencephalopathy (PML): Workshop Proceedings. 25-26 July 2011: European Medicines Agency. http://www.ema.europa.eu/docs/en_GB/doc ument_library/Report/2011/09/WC5001115 62.pdf. Accessed January 10, 2017.
23. Dreyer NA, Blackburn S, Mt-Isa S, et al. Direct-to-patient research: piloting a new approach to understanding drug safety during pregnancy. JMIR Public Health and Surveillance. 2015;2(1):e22.

24. Kiderlen M, Walsh PM, Bastiaannet E, et al. Treatment Strategies and Survival of Older Breast Cancer Patients - An International Comparison between the Netherlands and Ireland. PLoS One. 2015;10(2):e0118074. PMID: 25646756. PMCID: PMC4315587. Epub Feb 4.

25. Lutgens M, van Oijen M, Mooiweer E, et al. A risk-profiling approach for surveillance of inflammatory bowel disease-colorectal carcinoma is more cost-effective: a comparative cost-effectiveness analysis between international guidelines. Gastrointest Endosc. 2014 Nov;80(5):842-8. PMID: 25088918. Epub Aug 5.

26. Vrdoljak E, Wojtukiewicz MZ, Pienkowski $\mathrm{T}$, et al. Cancer epidemiology in Central, South and Eastern European countries. Croat Med J. 2011 Aug 15;52(4):478-87. PMID: 21853542. Epub Aug 20.

27. Ferlay J, Shin HR, Bray F, et al. Estimates of worldwide burden of cancer in 2008: GLOBOCAN 2008. Int J Cancer. 2010 Dec 15;127(12):2893-917. PMID: 21351269. Epub Feb 26.

28. Parkin DM, Bray F, Ferlay J, et al. Global cancer statistics, 2002. CA Cancer J Clin. 2005 Mar-Apr;55(2):74-108. PMID: 15761078. Epub March 12.

29. Wild D, Grove A, Martin M, et al. Principles of Good Practice for the Translation and Cultural Adaptation Process for PatientReported Outcomes (PRO) Measures: report of the ISPOR Task Force for Translation and Cultural Adaptation. Value Health. 2005 Mar-Apr;8(2):94-104. PMID: 15804318. 
30. European Medicines Agency. Guideline on good pharmacovigilance practices (GVP). Annex I - Definitions (Rev 3). 15 April 2014. http://www.ema.europa.eu/docs/en_GB/doc ument_library/Scientific_guideline/2013/05/ WC500143294.pdf. Accessed January 10, 2017.

31. Directive 95/EC of the European Parliament and of the Council on the Protection of Individuals with Regard to the Processing of Personal Data and on the Free Movement of Such Data. European Union, The Council: Brussels, 1995. http://europa.eu/legislation_summaries/infor mation_society/l14012_en.htm. Accessed January 10, 2017.

32. Department of Health and Human Services. Office of the Secretary. 45 CFR Parts 160 and 164. HIPAA Administrative Simplification: Enforcement; Final Rule. Federal Register Vol 71;32: Feb 16, 2006.

33. Dreyer NA, Blackburn S, Hliva V, et al. Balancing the Interests of Patient Data Protection and Medication Safety Monitoring in a Public-Private Partnership. JMIR Med Inform. 2015;3(2):e18.

34. Ispe. Guidelines for good pharmacoepidemiology practices (GPP). Pharmacoepidemiol Drug Saf. 2008 Feb;17(2):200-8. PMID: 17868186.
35. WMA Declaration of Helsinki - Ethical Principles for Medical Research Involving Human Subjects. October 2013. http://www.wma.net/en/30publications/10po licies/b3/. Accessed January 10, 2017.

36. U.S. Food and Drug Administration. Guidance for Industry. E6 Good Clinical Practice: Consolidated Guidance. April 1996. http://www.fda.gov/downloads/Drugs/Guida nces/ucm073122.pdf. Accessed January 10, 2017.

37. International Society for Pharmacoepidemiology. Data Privacy, Medical Record Confidentiality, and Research in the Interest of Public Health. 1 September 1997. Amended 19 August 1998.

https://www.pharmacoepi.org/resources/priv acy.cfm. Accessed January 10, 2017.

38. GARFIELD-AF Registry | Thrombosis Research Institute. http://www.trilondon.ac.uk/garfield. Accessed January 10, 2017.

39. Dreyer NA, Blackburn S, Mt-Isa S, et al. PROTECT Pregnancy Study: An Exploratory Study of Self-Reported Medication Use in Pregnant Women. Pharmacoepidemiological Research on Outcomes of Therapeutics by a European ConsorTium; 2015. 